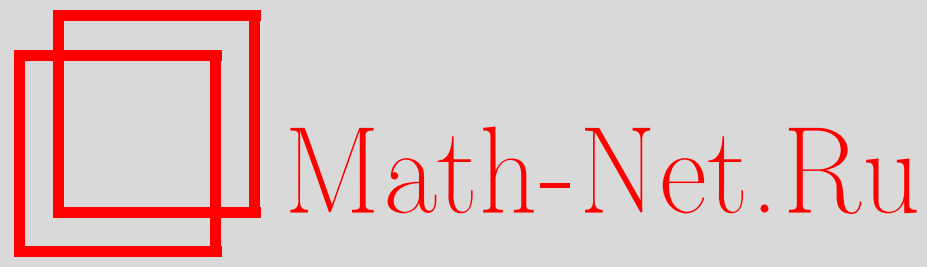

В. В. Козлов, Топология вещественных алгебраических кривых и интегрируемость геодезических потоков на алгебраических поверхностях, Функи. анализ и его прил., 2008, том 42, выпуск 2, 23-27

DOI: https://doi.org/10.4213/faa2899

Использование Общероссийского математического портала Math$\mathrm{Net.Ru}$ подразумевает, что вы прочитали и согласны с пользовательским соглашением http://www . mathnet.ru/rus/agreement

Параметры загрузки:

IP : 54.157 .27 .8

26 апреля 2023 г., 04:02:30

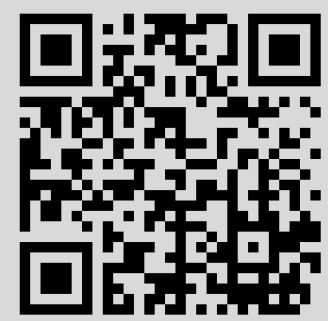


Функционалъный анализ и его приложения

2008, т. 42, вып. 2, с. 23-27

УДК $517.93+512.77$

\title{
Топология вещественных алгебраических кривых и интегрируемость геодезических потоков на алгебраических поверхностях
}

\author{
(c) 2008. В. В. Козлов
}

Рассматривается задача о наличии дополнительного первого интеграла уравнений геодезических на некомпактных алгебраических поверхностях. Она обсуждалась еще Риманом и Дарбу. Указаны грубые препятствия к интегрируемости, которые связаны с топологией вещественной алгебраической кривой линии пересечения такой поверхности со сферой большого радиуса. Отсутствие нетривиального первого интеграла геодезического потока на алгебраической поверхности со сложным топологическим строением вытекает из наличия бесконечного числа неустойчивых замкнутых геодезических, реализующих замкнутые циклы. Обсуждаются некоторые пока не решенные задачи.

Автор дружески благодарит А. В. Борисова и И. С. Мамаева, обративших его внимание на этот круг вопросов.

\section{§1. Геодезические потоки}

Пусть $E$ - трехмерное евклидово пространство с декартовыми координатами $\left(x_{1}, x_{2}, x_{3}\right)=x$ и $f$ - однородный ненулевой многочлен от $x$ степени $m$. Геодезический поток на алгебраической поверхности

$$
f(x)=c
$$

задается дифференциальным уравнением второго порядка

$$
\ddot{x}=\lambda \frac{\partial f}{\partial x} .
$$

Точка обозначает дифференцирование по переменной $t$, которая задает параметризацию геодезической. В механике уравнение (2) описывает движение частицы единичной массы по гладкой поверхности (1); параметром $t$ служит время.

Множитель Лагранжа $\lambda$ находится явно с учетом уравнения связи (1):

$$
\lambda=-\frac{(A \dot{x}, \dot{x})}{\left(f^{\prime}, f^{\prime}\right)}
$$

где $($, ) - скалярное произведение, определяемое евклидовой структурой пространства $E$,

$$
A=\left\|\frac{\partial^{2} f}{\partial x_{i} \partial x_{j}}\right\|
$$

- матрица Якоби, $f^{\prime}=\partial f / \partial x$. По формуле Эйлера $c=0$ - единственное критическое значение функции $f$. Поэтому при $c \neq 0$ знаменатель в (3) не 
обращается в нуль на поверхности (1). Если $f^{\prime}(x)=0$ лишь при $x=0$, то уравнение (2) с учетом формулы (3) не имеет сингулярностей на конусе

$$
\{f(x)=0, x \neq 0\} .
$$

Уравнение (2) допускает очевидный интеграл энергии

$$
H=\frac{(\dot{x}, \dot{x})}{2} .
$$

Если имеется дополнительный независимый первый интеграл, то задача о геодезических вполне интегрируема. Задача о геодезических на квадриках $(m=2)$ в многомерном случае решена в классических работах Якоби и Шаля. Их современное прочтение содержится в добавлении 14 к книге В. И. Арнольда [1]. Дополнительные интегралы - однородные многочлены по скоростям второй степени.

Мы рассматриваем эту задачу при $m \geqslant 3$. Она встречается, например, в работе Римана [2] при исследовании движения жидкого однородного эллипсоида. Более точно, у Римана речь идет об интегрируемости геодезического потока на поверхности третьего порядка

$$
x_{1} x_{2} x_{3}=\text { const. }
$$

Отметим сначала, что задача о геодезических на конусе (4) вполне интегрируема при всех значениях $m$. Имеется дополнительный независимый интеграл, квадратичный по скорости:

$$
\Phi=([x, \dot{x}],[x, \dot{x}]) .
$$

Здесь [ , ] - операция векторного умножения. Функция (6) имеет смысл квадрата кинетического момента частицы относительно начала координат. В этом случае несложно ввести разделяющиеся переменные на конусе (4) и свести задачу о геодезических к квадратурам.

\section{§2. Условия неинтегрируемости}

При $c \neq 0$ интегрируемость геодезического потока на алгебраической поверхности $\{f(x)=c\}$, по-видимому, является редким исключением. В дальнейшем предполагается, что

$$
f^{\prime}(x) \neq 0 \quad \text { при } x \neq 0
$$

и что конус $\{f(x)=0\}$ не сводится к одной точке - началу координат $x=0$. В этом случае конус пересекается с единичной сферой

$$
x_{1}^{2}+x_{2}^{2}+x_{3}^{2}=1
$$

по заведомо регулярной (в вещественном смысле) алгебраической кривой $F$. Она состоит из нескольких непересекающихся овалов. Рассмотрим еще область $F_{+}$на сфере $(8)$, заданную неравенством $f(x) \geqslant 0$. Эта область топологически устроена как объединение дисков с дырками.

Основной результат состоит в следующем.

Теорема. Если эйлерова характеристика области $F_{+}$отрицательна, то ни при каких с >0 геодезический поток на поверхности (1) не допускает первого интеграла, аналитического по координатам и скоростям и независимого от интеграла энергии $H$. 
Алгебраическая поверхность, конечно, может оказаться несвязной. Теорему следует понимать так: хотя бы на одной из связных компонент поверхности (1) геодезический поток будет неинтегрируемой динамической системой. Формулировку теоремы можно слегка усилить: одна из связных компонент области $F_{+}-$ это диск с $p \geqslant 2$ дырками.

Аналогичный результат справедлив и для $c<0$ (только область $F_{+}$надо заменить на $F_{-}$, где $\left.f(x) \leqslant 0\right)$. Таким образом, свойство интегрируемости геодезического потока зависит от топологии вещественных алгебраических кривых на сфере (или на проективной плоскости, если отождествить антиподальные точки). Обсудим теперь условия теоремы.

А. Пусть $f$ - однородный многочлен от $x_{3}$ и $x_{1}^{2}+x_{2}^{2}$. Тогда алгебраическая поверхность (1) будет поверхностью вращения и уравнение геодезических допускает интеграл, линейный по скоростям: $\Phi=x_{1} \dot{x}_{2}-\dot{x}_{1} x_{2}$. В этом случае связные компоненты области $F_{+}$будут либо дисками, либо кольцами. В первом случае $\chi=1$, а во втором $\chi=0$. Таким образом, условия теоремы заведомо не выполнены.

В. Если $m=2$, то дополнение к алгебраической кривой $F$ на сфере в типичном случае состоит из двух дисков и одного кольца, а при вырождении из двух дисков.

C. При $m=3$ условие теоремы также не выполнено. Дело в том, что в этом случае алгебраическая кривая $F$ может иметь не более трех связных компонент (неравенство Харнака), причем один овал инвариантен при инволюции $x \mapsto-x$, а два других переходят при таком отображении друг в друга. Поэтому топологическое строение связных компонент области $F_{+}$такое же, как и в примерах $\mathrm{A}$ и В.

D. Пусть $m=4$ и

$$
f=\left(x_{1}^{2}-x_{2}^{2}-x_{3}^{2}\right)\left(x_{2}^{2}-x_{1}^{2}-x_{3}^{2}\right) .
$$

Хотя в этом случае условие (7) не выполнено, тем не менее алгебраическая кривая $F$ есть объединение четырех непересекающихся окружностей. Поскольку множество $F_{+}$- диск с тремя дырками, то геодезический поток на поверхности четвертого порядка $f=c>0$ не будет интегрируемой динамической системой.

Е. Алгебраическая кривая $F$ инвариантна при инволюции $x \mapsto-x$. Следовательно, ее можно рассматривать на проективной плоскости $\mathbb{R} P^{2}$, которая получается из единичной сферы (8) отождествлением антиподальных точек. В частности, $M$-кривые Харнака отвечают алгебраическим поверхностям, удовлетворяющим условиям теоремы (для $c>0$ или $c<0$ ) при всех $m \geqslant 4$. Таким образом, для любого целого $m \geqslant 4$ найдется алгебраическая поверхность степени $m$ с неинтегрируемым геодезическим потоком, причем свойство неинтегрируемости сохранится при малом шевелении коэффициентов многочлена $f$.

Оценки и сравнения для $\chi\left(F_{+}\right)$- предмет известных работ по вещественной алгебраической геометрии (см. обзоры [3], [4]). Однако результаты этих работ мало что дают нам относительно условий интегрируемости геодезических потоков (поскольку они носят, как правило, общий характер, а с другой стороны, при каждом $m \geqslant 2$ имеется алгебраическая поверхность степени $m$ с интегрируемым потоком). Отметим только одно следствие известного сравнения Гудкова: 
если $F$ является $M$-кривой (т. е. кривой, имеющей максимально возможное число связных компонент) и $m=4+8 k, k \geqslant 0$ целое, то геодезический поток на хотя бы одной связной компоненте соответствующей алгебраической поверхности будет неинтегрируемым.

\section{§3. Доказательство основной теоремы}

Доказательство неинтегрируемости основано на наличии бесконечного числа неустойчивых (в типичном случае гиперболических) периодических геодезических, в точках которых первые интегралы зависимы. Эти замкнутые траектории расположены в фазовом пространстве достаточно сложным образом, что и препятствует существованию дополнительного интеграла. Для компактных поверхностей общая теорема о топологических препятствиях к интегрируемости установлена в [5]. Ее вариант для многообразий с геодезически выпуклым краем содержится в работе Болотина [6] (с приложениями к задачам небесной механики). Формальное доказательство основной теоремы выводится из общих результатов статьи [6].

Рассмотрим кусок алгебраической поверхности (1), пересекающийся со сферой большого радиуса:

$$
M_{c, r}=\{x \in E: f(x)=c,|x| \leqslant r\} .
$$

Покажем, что его граница геодезически выпукла: если геодезическая касается границы, то в проколотой окрестности точки касания она лежит вне $M_{c, r}$. Итак, пусть $t \mapsto x(t)$ - геодезическая и $(x, \dot{x})=0$ в момент времени $t=0$ в некоторой точке $x_{0}\left(f\left(x_{0}\right)=c,\left|x_{0}\right|=r\right)$. Вычислим вторую производную расстояния:

$$
(x, x)^{\cdot *}=2(x, \dot{x})^{\cdot}=2(x, \ddot{x})+2(\dot{x}, \dot{x})=2(\dot{x}, \dot{x})-\frac{2 m c(A \dot{x}, \dot{x})}{\left(f^{\prime}, f^{\prime}\right)} .
$$

Согласно предположению, $\left(f^{\prime}, f^{\prime}\right) \neq 0$ при всех $x \neq 0$. Далее, при фиксированном $c$ коэффициенты второй квадратичной формы в (9) - однородные функции от $x$ степени $-m$. Следовательно, они стремятся к нулю при $|x| \rightarrow \infty$. Но это означает, что квадратичная форма (9) положительно определена, если $|x|$ достаточно велико. Таким образом, при $t=0$

$$
(x, x)^{\cdot}=2(x, \dot{x})=0, \quad \text { a }(x, x)^{*}>0 .
$$

Но это означает, что $|x(t)|>\left|x_{0}\right|$ при малых $|t| \neq 0$.

Заметим теперь, что $\chi\left(M_{c, r}\right)<0$, если $r$ достаточно велико. Действительно, согласно предположению теоремы, $\chi\left(F_{+}\right)<0$. Тогда граница связной области $F_{+}$состоит из $p \geqslant 3$ овалов. По непрерывности граница многообразия $M_{c, r}$ при малых $c>0$ и $r=1$ также содержит $p$ овалов. После преобразования подобия $x \mapsto \lambda x$ с подходящим коэффициентом $\lambda>1$ из $M_{c, 1}$ получаем нужное нам многообразие с краем $M_{c, r}$ (конечно, при этом $\left.c \mapsto \lambda^{m} c\right)$. Из элементарной топологии двумерных поверхностей отсюда сразу следует отрицательность эйлеровой характеристики многообразия $M_{c, r}$.

Таким образом, одна из связных компонент алгебраической поверхности (1) имеет вложенный геодезически выпуклый кусок с отрицательной эйлеровой характеристикой. Неинтегрируемость соответствующего геодезического потока вытекает теперь из общего результата работы [6]. 


\section{§4. Некоторые задачи}

В заключение отметим некоторые пока не решенные задачи.

(а) Доказать неинтегрируемость уравнений геодезических на конусе $\{f=0\}$ в многомерном случае. Напомним, что для полной интегрируемости геодезического потока на $n$-мерном многообразии требуется $n$ независимых инволютивных первых интегралов.

(b) Верно ли, что геодезический поток на алгебраической поверхности третьего порядка общего вида неинтегрируем? Хорошо бы дать строгое доказательство неинтегрируемости для поверхности (5).

(c) Геодезический поток на алгебраической поверхности (1) определяется только самой однородной формой $f$, т. е. набором ее коэффициентов - точкой аффинного пространства (ввиду инвариантности уравнений геодезических при замене $f$ на $\lambda f, \lambda \neq 0$, аффинное пространство коэффициентов можно заменить проективным пространством). Вопрос состоит в том, являются ли условия интегрируемости геодезического потока алгебраическими, т. е. задаются ли они конечным числом алгебраических уравнений на коэффициенты однородной формы $f$ ?

(d) Ввиду квадратичности правых частей уравнений геодезических по скоростям наличие дополнительного аналитического интеграла эквивалентно наличию интеграла в виде однородного многочлена по скоростям, независимого от интеграла энергии. Например, интегралы из 1 были линейными или квадратичными по скоростям. Дополнительный интеграл минимально возможной степени назовем неприводимым. Спрашивается, имеются ли алгебраические поверхности, геодезические потоки которых допускают неприводимые интегралы сколь угодно высокой степени?

(е) Следовало бы найти ограничения на топологию алгебраических многообразий произвольной размерности $n$ с вполне интегрируемыми геодезическими потоками. Верно ли, что первое число Бетти таких многообразий не превосходит $n$ ? Для компактности многообразий (не обязательно алгебраических) топологические ограничения указаны Таймановым [7].

\section{ЛитерАтУРА}

[1] В. И. Арнольд, Математические методь классической механики, Эдиториал УРCC, М., 2000.

[2] Б. Риман, О движении жидкого однородного эллипсоида, в кн.: Сочинения, ОГИЗ, М., Л., 1948, 339-366.

[3] Д. А. Гудков, Топология вещественных проективных алгебраических многообразий, УМН., 29:4 (1974), 3-79.

[4] В. А. Рохлин, Комплексные топологические характеристики вещественных алгебраических кривых, УМН., 33:5 (1978), 77-89.

[5] В. В. Козлов, Топологические препятствия $к$ интегрируемости натуралъных механических систем, ДАН СССР, 249:6 (1979), 1299-1302.

[6] С. В. Болотин, Неинтегрируемость задачи $n$ иентров при $n>2$, Вестн. Моск. ун-та, сер. матем., механ., 1984, № 3, 65-68.

[7] И. А. Тайманов, Топологические препятствия $\kappa$ интегрируемости геодезических потоков на неодносвязных многообразиях, Изв. АН СССР, сер. матем., 51:2 (1987), 429-435.

Математический институт им. В. А. Стеклова e-mail: vvkozlov@mi.ras.ru 